\title{
Children use inverse planning to detect social transmission in design of artifacts
}

\author{
Madison L. Pesowski (mpesowski@ucsd.edu), \\ Alyssa D.Quy (aquy@ucsd.edu), \\ Michelle Lee (m.lee@ucsd.edu), \\ Adena Schachner (schachner@ucsd.edu) \\ University of California, San Diego, Department of Psychology \\ 9500 Gilman Drive, San Diego, CA 92093-0109 USA
}

\begin{abstract}
Do children use objects to infer the people and actions that created them? We ask how children judge whether designs were socially transmitted (copied), asking if children use a simple perceptual heuristic (more similar $=$ more likely copied), or make a rational, flexible inference (Bayesian inverse planning). We found evidence that children use inverse planning to reason about artifacts' designs: When children saw two identical designs, they did not always infer copying occurred. Instead, similarity was weaker evidence of copying when an alternative explanation 'explained away' the similarity. Thus, children inferred copying had occurred less often when designs were efficient (Exp1, age 7-9; $\mathrm{N}=52$ ), and when there was a constraint that limited the number of possible designs (Exp2, age 4-5; $\mathrm{N}=160$ ). When thinking about artifacts, young children go beyond perceptual features and use a process like inverse planning to reason about the generative processes involved in design.
\end{abstract}

Keywords: social cognition; cognitive development; Bayesian inference; inverse planning; artifact design

\section{Introduction}

Children around the world grow up surrounded by humanmade objects, or artifacts. These objects are important not only because they serve functions (e.g., a cup exists to hold liquid) but because they convey a vast amount of social information. Objects tell us about the people who own or created them, and allow for quick and accurate judgements of others' group affiliations, traits, and interests (Gosling, 2008; Richins, 1994). This reasoning has been termed intuitive archeology-like archeologists, people use objects to learn about the people who created them (Hurwitz, Brady, Schachner, 2019; Schachner et al., 2018).

How do children reason about others from the objects they create? Here we build on work characterizing the cognitive basis of this reasoning in adults, and ask how such reasoning develops in childhood. We test the hypothesis that children, like adults, make rational social-causal inferences from artifacts - by integrating their mental theories of the physicalmechanical world with their theories of the social world (e.g. Gopnik, 2012; Liu et al., 2017; Spelke \& Kinzler, 2007).

To model and test the nature of children's reasoning, we focus on a foundational inference in this domain: Inferring whether a design idea was socially transmitted through imitation or copying; or whether the design idea was generated independently by an individual. These two basic processes - imitation and innovation - form the basis for cultural evolution of artifacts over human history (Henrich, 2017; Legare \& Nielsen, 2015; Tomasello, 1999). In the domain of social reasoning, this inference provides a foundation for inferring a rich array of other social information: Designs that were copied license different inferences than those that were independently generated. For example, a copied design may provide information about the creator's social history and cultural group (Soley \& Spelke, 2016); a design that was independently generated may hold information about creativity or intelligence (Gosling, 2008).

\section{Children's reasoning about copying in design}

For children, copying is salient and socially relevant from early in life: Children use copying to infer social affiliation (Over \& Carpenter, 2015), dislike plagiarizers (Olson \& Shaw, 2011), and engage in social and cultural learning (Henrich, 2017). We thus ask: How do children make the inference that copying has occurred? Two alternative types of cognitive processes may underlie children's reasoning.

Firstly, children may detect copying using a simple rule: If designs look more similar, then they are more likely copied. This approach relies on a simple heuristic, based solely on salient perceptual features - a strategy often seen in young children (e.g., Piaget, 1929).

In contrast, we hypothesize that children make inferences that go beyond perceptual features, and instead make rational inferences using inverse planning (e.g., Baker, Saxe, \& Tenenbaum, 2009). Children's detection of copying thus provides a test case for a broader question: Can children reason about how objects were generated, using the features of objects to infer what caused them to be built that way?

\section{Inverse planning about artifacts}

Past work has shown that children and adults use inverse planning to reason about others' actions, allowing them to infer goals, preferences, and beliefs from the movements they observe (Baker et al., 2009; 2017; Jara-Ettinger et al., 2016; Liu et al., 2017). This reasoning has been modeled as a form of rational Bayesian inference to the best explanation (Lipton, 2004; Tenenbaum, Griffiths, \& Kemp, 2006): From early in life, people have a mental model of rational planning, which allows them to predict what a rational agent would do, given a goal and any environmental constraints (Gergely et al., 1995; Jara-Ettinger et al., 2016; Liu et al., 2017). The idea of inverse planning is that people reason about others' actions 
by inverting this generative process, using people's behaviors to infer their goals and constraints.

We propose that adults and children use a fundamentally similar inverse planning process to reason about the artifacts people create. Just like for actions, when we observe artifacts - the products of actions - we may be able to reason about the goals and constraints that would lead a rational agent to build something with those features. Recent work has provided evidence that adults use inverse planning to reason about the source of design ideas in this way (Hurwitz et al., 2019; Schachner et al., 2018).

If children use inverse planning to reason about artifacts, then they should be able to make rich and flexible inferences about the source of design ideas (e.g., copying), taking into account others' goals and environmental constraints. This account thus makes specific predictions about copying detection: When children see two identical artifacts, they should not infer that copying has occurred equally often in all cases. Instead, alternative explanations for why two people created similar artifacts, such as an independently-shared bias (e.g., the tendency to create efficient designs) or a functional constraint (e.g., a barrier limiting the number of designs that would work), should 'explain away' similarity, making similarity weaker evidence of copying.

Prior work suggests that children may have the cognitive prerequisites for this kind of social-causal reasoning about objects. By age 3, children understand that object features typically serve functions, and by age 4 , expect people to create efficient tools (Kelemen, Seston, \& Saint Georges, 2012). By age 4, children are also able to think about objects' histories when judging which objects people care about (Pesowski \& Friedman, 2019), and categorize objects in terms of their intended function, not just their perceptual features (Bloom \& Markson, 1998; Diesendruck, Markson, \& Bloom, 2003; Gelman \& Bloom, 2000).

In the current work, we test whether children use inverse planning to reason about objects' designs, using copying detection as a case study to tease apart the inverse planning account from a simpler cognitive mechanism (perceptual heuristics). In two experiments, we ask whether children make rational inferences about whether a design was copied, flexibly taking into account two different kinds of alternative explanations for similarity in making their judgments: efficiency (Exp. 1) and functional constraints (Exp. 2).

The data, analysis code, and experimenter scripts for both experiments are available at https://osf.io/zxw6e/.

\section{Experiment 1}

In a first experiment, we ask whether 7-9-year-old children use inverse planning or a perceptual heuristic when detecting copying from artifacts' designs. If children use inverse planning, they should expect that others will act rationally by building efficient designs (Baker et al., 2009; 2017). People generally have a strong, independent desire to build efficient designs, which constrains the designs they are likely to build (Dennett, 1990). Efficiency should thus serve as an alternative explanation for similarity, 'explaining away' the similarity and making it weaker evidence of copying.

To test this account, we introduced children to a train-track building task, modeled after adult work using a similar method (Schachner et al., 2018). In this task, the goal is to build a track to get a train from one location to another location quickly, using puzzle-like pieces on a game board grid. On each trial, children viewed a video involving two characters, and then saw a pair of train tracks that the two characters built. Based on their designs, children were asked to judge whether one of the characters had copied the other, or whether they had created the designs independently.

We manipulated the efficiency of the tracks in two ways. First, we manipulated the length of the tracks: Shorter tracks are more efficient than longer tracks, and thus identical shorter tracks should be judged as less likely copied than identical longer tracks. Second, we introduced a barrier to the game board that the tracks could not go through. In the context of the barrier, track designs that were formerly extremely inefficient now become the most efficient possible design (see Figure 1). Lastly, we also separately manipulated the tracks' level of perceptual similarity (i.e., whether they are the same or different).

If children use inverse planning to reason about the source of artifacts' designs, then efficiency should 'explain away' similarity, making it weaker evidence of copying. Children should thus judge that copying occurred less often for efficient designs than inefficient designs - even if the designs are perceptually identical in both cases. In contrast, if children use a heuristic based on perceptual similarity, they should infer that all identical designs are equally likely copied, and efficiency should not affect their judgements.

\section{Methods}

Participants. 52 7-9-year-olds participated ( $M_{\text {age }}=8$ years; 8 months, range $=7 ; 8-9 ; 11,25$ males). Children were recruited from the metro San Diego area from a database of local families interested in research. An additional 5 children were tested but excluded due to technical error.

Materials and Procedure. Participants were tested individually, seated across from an experimenter.

Building Phase. Participants were shown a 9x9 game board grid with two houses (see Figure 1). Children were given 7 straight track pieces and 7 curved pieces, and asked to build a track on the board "to get the train from one house to the other house in the fastest, quickest way". If children failed to connect the houses, they were prompted to correct their track. To check children's comprehension of efficiency, they were shown two pairs of tracks and asked, "Which track is faster?". If children responded incorrectly, they were asked, "Which track is shorter?". All but one child answered the first question correctly; all answered at least one correctly.

Test Trials. Using a within-subject design, children completed 8 unique test trials. On each trial, children saw a video of a pair of puppets on an iPad. In each video, the 


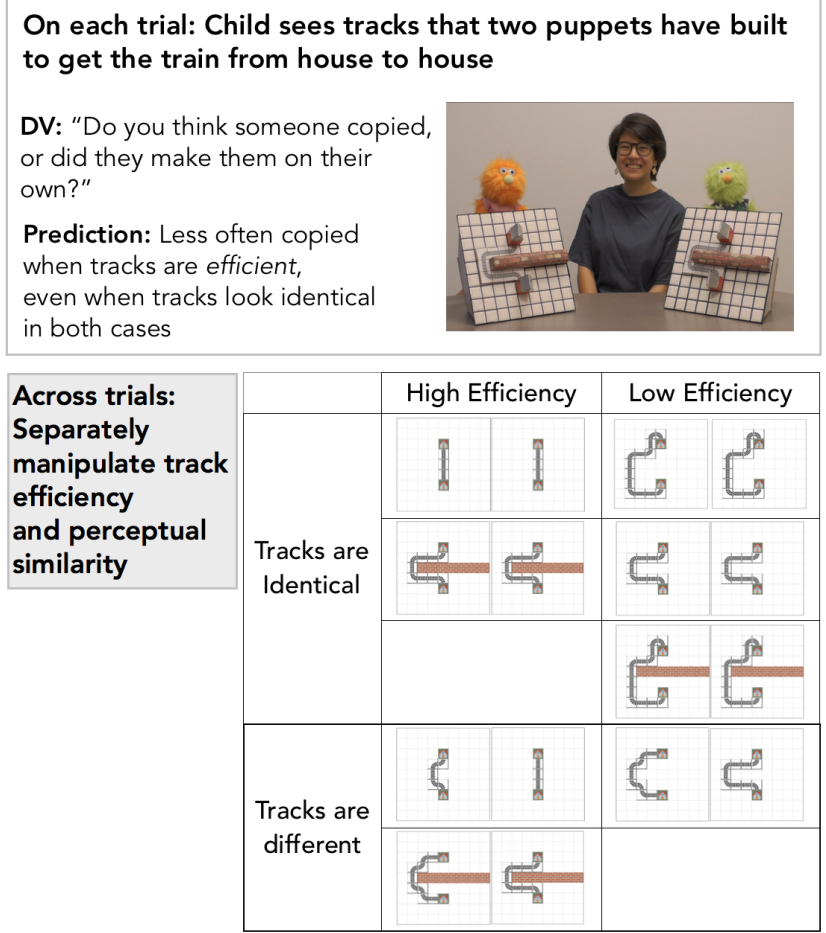

Figure 1: Exp. 1, Method. Children saw pairs of track designs, and judged if copying had occurred. To test whether children would judge similarity to be weaker evidence of copying when the design was highly efficient, we separately manipulated the tracks' efficiency and perceptual similarity across trials.

puppets were introduced, and each given identical game boards and sets of train track pieces. Participants were told that puppets were asked "to build a train track that gets the train from one house to the other, in the fastest, quickest way". The tracks the puppets built were then shown (see Figure 1). Children were asked: "Do you think someone copied, or do you think they made them on their own?", and asked to explain their answer ("What makes you think that?"). Different pairs of puppets were shown in each trial, distinguished by color, clothing, and names.

Design and Logic. Across trials, we manipulated the tracks' perceptual similarity (same/different), and efficiency (high/low; see Figure 1). The order of trials was pseudorandomized across participants, with trials in one of four orders. A building phase was always presented first, followed by two test trials, the other building phase, and then the six remaining test trials. Whether the first building phase involved the game board with or without the barrier was counterbalanced across participants.

\section{Results}

To ask whether children consider the tracks' efficiency, not just perceptual similarity, we used a logistic regression model, which predicted copying judgements based on four predictors: The perceptual similarity of the tracks

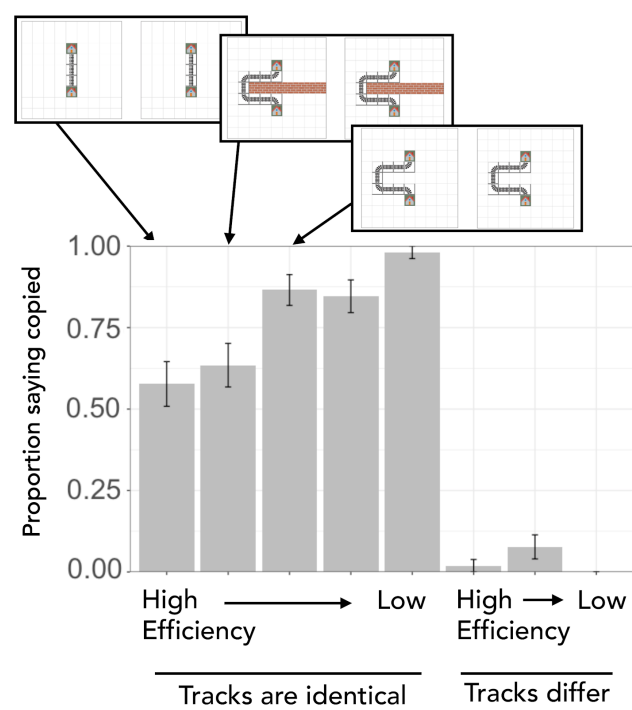

Figure 2: Exp. 1, Results. Proportion of children saying copying occurred (Y-axis), for each test trial, sorted by perceptual similarity and efficiency (X-axis). Error bars are standard error. 7-9-year-old children judged identical efficient tracks as less likely copied than identical inefficient tracks. When detecting copying, children go beyond how similar two designs look and consider alternative explanations for similarity, like the tendency to create efficient designs.

(same/different), the efficiency of the tracks (high/low), participants' age (in months), and subject (as a random factor). In line with the use of inverse planning, we found that efficiency significantly improved model fit, above and beyond the effects of perceptual similarity and the other factors (nested logistic model comparison, $\chi^{2}(1)=24.87$; $p<0.0001)$. Children judged that tracks were copied more often when they were inefficient $(M=67 \%, \mathrm{SEM}=2.1 \%)$ than efficient $(M=33 \%$, SEM=2.8\%).

Efficiency predicted copying judgements even when comparing across pairs of equally similar tracks, and even when the exact same design was built in two contexts that made it inefficient vs. efficient (No barrier vs. Barrier; Figure 2). Perceptual similarity also impacted children's judgements of copying, as predicted by both inverse planning and perceptual heuristic accounts (nested logistic model comparison, $\left.\chi^{2}(1)=236.57 ; p<0.0001\right)$. There was no effect of age: Age did not improve model fit beyond the other factors (nested logistic model comparison, $\chi^{2}(1)=2.22 ; p=0.136$ ). Overall these data provide evidence that by age 7 , children use inverse planning to reason about artifacts' designs.

\section{Experiment 2}

In Exp. 1, we found that children took into account the efficiency of the design when judging whether copying had occurred, judging similarity to be weaker evidence of copying when the design was highly efficient. This suggests that by age 7 , children use inverse planning to reason about artifacts' designs. However, there is still an alternative 
possibility, which we test here. It is possible (and even plausible) that efficiency is unique and privileged in children's reasoning. Reasoning about efficiency is thought to be foundational to cognition: It develops early in infancy (Gergely et al., 1995; Skerry, Carey \& Spelke, 2013), is shared with other species (Hauser \& Wood, 2010); and is a foundation for the entire domain of action understanding (Baker et al., 2017; Dennett, 1987). The evidence thus far is consistent with a simpler system than a complete inverse planning account: Children may be able to understand the role of efficiency in artifacts' designs, without flexibly taking into account a wider variety of alternative explanations.

In Exp. 2, we had two goals. First, we aimed to test whether children's reasoning was flexible, or limited to efficiencybased reasoning. To do this, we asked whether children rationally consider functional constraints: Whether each of the possible designs would function. A stricter set of functional requirements can constrain the set of possible solutions, making similar designs less of a suspicious coincidence (Tenenbaum \& Griffiths, 2001). Rationally speaking, since clearly non-functional designs are unlikely to be built, if many possible designs would work to solve the problem, similarity should be stronger evidence of copying than if fewer options would work.

Second, we asked whether inverse planning was possible even earlier in development than age 7-9. We thus tested younger children, age 4-5 years, in Exp. 2.

To test our predictions, we use a simple artifact-building task to manipulate functional constraints. Children were asked to solve one of two puzzle boxes, by building a tool to reach a button inside. Across between-subject conditions, the two boxes differed in one respect: How many designs would work to solve them. For one box, all 10 possible tool designs would solve the puzzle (unconstrained, circle-box). For the other box, only 1 of the 10 possible designs would work (constrained, star-box). This box thus introduced a constraint that limited the set of functional designs. Children were asked to build a tool to solve the puzzle; and a puppet was given the same task, with an identical set of pieces. Crucially, the puppet always built the same design as the child. The child was then asked to infer: Did he copy you, or did he think of that on his own?

If children use flexible inverse planning to reason about designs, children should treat functional constraints as an alternative explanation for the design similarity. Thus, the presence of a functional constraint should explain away the tools' similarity, and children should judge that copying occurred less often in the constrained (star-box) condition than the unconstrained (circle-box) condition - even though the tools created are identical in both cases. If children use a heuristic based on perceptual similarity, or consider only efficiency, then they should say copying occurred equally often across the two conditions.

\section{Methods}

Participants. 160 4-5-year-olds $\left(M_{\mathrm{age}}=4 ; 9\right.$, range $=4 ; 0-5 ; 11$, 80 males) were recruited from the metro San Diego area. An additional 34 children were tested but excluded because they did not respond to the main test question, said "I don't know", or provided an ambiguous response (e.g., "On his own with me") (7); responded incorrectly to a memory question in the test trial (16); refused to finish participating (3); had parental interference (1); birthdate was not provided, preventing age calculation (1); or experienced technical issues (6).

Logic. Two between-subject conditions manipulated functional constraints (see Figure 3). In the circle-box condition, the hole in the top of the box was circular, and tool designs were unconstrained: All 10 rod options fit into the puzzle box and worked to solve the puzzle (note: there was no circle-shaped rod). In the star-box condition, tool designs were highly constrained: Only 1 rod (the star-shaped rod) fit into the puzzle box and worked to solve the puzzle.

Materials and Procedure. Warm up. To familiarize children with the experimenter and the nature of the task (i.e., to make judgments about copying), children were first told two stories in which two agents drew identical pictures and were asked to indicate whether they thought one agent had copied the other. For example, in one story, children were told that a teacher taught her class about octopi, and two students subsequently drew pictures of an octopus. Following this, children were asked: "Do you think [character 1] copied [character 2], or do you think that [1] came up with that on her own?". Importantly, these warm-up stories did not involve any functional constraints.

Test trial. Children were introduced to a puppet ("Cookie Monster") and were both shown a puzzle: A button inside a box, which had a glass front and only a small hole in the top allowing access. Children were shown that they could not reach the button with their fingers, and children and the puppet were then each asked to build a tool to reach the button. To do so, the child and the puppet were each given an identical set of pieces (a handle and an identical set of 10 different-shaped rods); each chose one of the rods from their set and connected it to their handle. Children were not explicitly told if any or all of the rods fit into the box, and were allowed to freely decide which rod to use in their design. To maintain ambiguity about whether the puppet was copying the child's design, the puppet moved away from the table and faced away from the child while building.

The child always finished building first; when the child had finished, the experimenter commented on the child's choice ("you chose the $[\mathrm{X}]$ shape!"), to establish that the puppet knew what the child had built, and thus that copying was possible. Children were allowed to test whether their design worked (by fitting it through the hole in the box); if it did not work, children were allowed to try again (by choosing and connecting a different rod piece).

In all cases, the puppet built an identical tool to the child. Children were then asked: "Do you think Cookie Monster copied you, or did he think of that on his own?", followed by two memory check questions (who built each tool; whether each tool worked). 

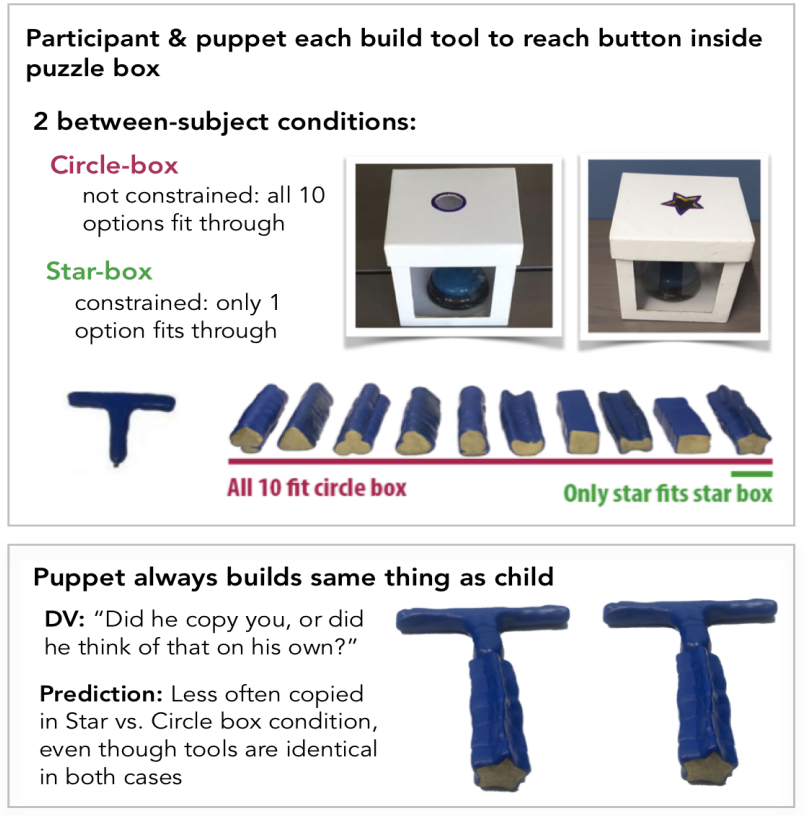

Figure 3: Exp. 2, Method. We manipulated functional constraints on the builders' designs and asked if children took these constraints into account when judging the likelihood of copying.

\section{Results}

To ask whether children consider functional constraints, not just perceptual similarity of the designs, we used a logistic regression model to predict judgments that copying had occurred, based on two predictors: Whether there was a functional constraint (puzzle-box condition: Star vs Circle), and participants' age (in months).

In line with the use of inverse planning, we found that copying judgments were affected by the presence of functional constraints (nested logistic model comparison, $\left.\chi^{2}(1)=13.3 ; p<0.001\right)$. In particular, children judged that the designs were copied more often when there was no constraint (Circle-box, 68.75\%) than when there was a functional constraint (Star-box, 40.0\%; see Figure 4). Age was also a significant predictor, such that across both conditions, older children were more likely to say that copying occurred than younger children $\left(\chi^{2}(1)=3.9 ; p=.048\right)$. Together with Exp. 1, these results show that children flexibly consider multiple alternative explanations for similarity, not just efficiency. Secondly, they show that preschool-age children also use inverse planning to reason about artifacts' designs.

\section{General Discussion}

In two experiments, we find evidence that children use a flexible inferential reasoning process - inverse planning - to reason about the source of design ideas. When reasoning about whether designs had been copied or generated independently, children did not always rely on how similar the two objects looked (their perceptual similarity). Instead, children took into account two kinds of alternative a.

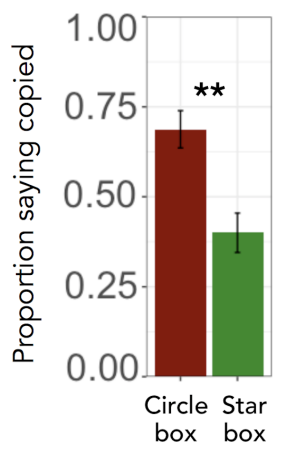

b.

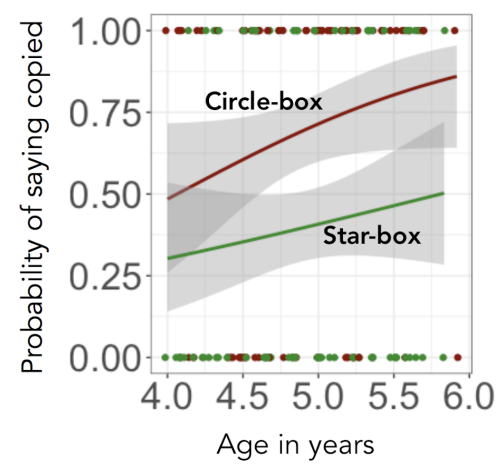

Figure 4: Exp. 2, Results. (a) Proportion of children saying copying occurred in each condition, error bars are standard error; (b) Probability of saying copying occurred, as a function of age and condition (logistic regression). Circle-box = not constrained; Star-box= constrained. 4- and 5 -year-old children judged constrained designs as less likely copied than unconstrained designs, even though the designs were identical in both cases. When detecting copying, 4-5year-olds go beyond how similar two designs look and consider alternative explanations for similarity, like functional constraints.

explanations for similarity: The tendency to create efficient designs (Exp. 1) and the presence of constraints limiting the number of functional designs (Exp. 2).

In both experiments, children judged artifacts' similarity to be weaker evidence of copying when an alternative explanation was available. This type of 'explaining away' is a signature of structured rational inference using a Bayes net (Gopnik \& Sobel, 2000): A given design can either be copied or independently generated, and evidence for one provides evidence against the other. Thus, if two people create identical designs, but this design is also likely to be created independently, this should provide weaker evidence of copying despite the identical designs.

In order to consider these alternative explanations, children must consider the generative processes behind artifact design - how and why the artifact was built. Thus, this reasoning is well-characterized as a form of inverse planning, in which reasoners invert their understanding of the generative process to make inferences about the goals and constraints that generated others' behavior. In line with this, prior work with adults has shown that formal Bayesian models of inverse planning best predict adults' copying judgments on similar tasks, which follow similar patterns to children's judgements reported here (Hurwitz et al., 2019; Schachner et al., 2018).

This work thus shows for the first time that children use inverse planning not only to reason about others' actions, but also to reason about physical objects that are the products of actions. In doing so, we provide a clear account of the cognitive basis of intuitive archeological reasoning: Through inverse planning, the physical objects we choose or create 
become tightly linked to actions, preferences, and goals -- and thereby to the social world.

Our findings are also informative about when, and under what circumstances, children will engage in inverse planning to reason about the source of design ideas. We find that by age 7, children consider efficiency as an alternative explanation for artifacts' similarity (Exp. 1), and by age 4, consider functional constraints (Exp. 2). We hypothesize that even preschool children have a broad ability to reason about both efficiency and functional constraints via inverse planning - but may show this competence only when the task is simple, and other cognitive requirements are minimized.

For example, in Experiment 2, children only needed to consider the actions and mental states of one other person besides themselves, while in Experiment 1 children made third-party judgments about two agents. Young children are known to be more adept at thinking about the knowledge state of a person interacting with them than third parties (Harris, Yang, \& Cui, 2017). In addition, thinking about design constraints may be easier when the result is binary (a design either works completely, or not at all, as in Exp. 2), versus when the design's quality is graded or continuous in nature (the train tracks in Exp. 1). Thus, while we find that young children can use inverse planning to reason about designs, with age children may become more able to spontaneously reason about the source of design ideas - even in complex, real-world situations. This hypothesis remains to be tested in future work.

We did find one age-related difference in Experiment 2 older children (5 years) were more likely than younger children to say that copying occurred, across all conditions. In a Bayesian framework, this may be formalized as a higher prior on copying in older children. Why would this occur? This could reflect an increased salience of copying to children after they enter school: Consistent with this, the CHILDES database shows that mentions of the word "copied" increase between ages 4 and 5, and mentions of "copy" peak at age 6 (result retrieved using http://childfreq.sumsar.net). Alternatively, it could reflect older children's greater tendency to copy: Exact or faithful imitation of others' behavior is known to increase with age (Marsh, Ropar, \& Hamilton, 2014; McGuigan \& Whiten, 2009; McGuigan et al., 2007; Nielsen \& Tomaselli, 2010). This account fits well with an inverse planning framework, as by this account children use their own increased tendency to copy to inform judgements about others' behaviors.

In conclusion, the question of whether children's reasoning is best characterized as driven by perceptual biases and heuristics or by flexible, abstract reasoning is a core debate in cognitive development that dates back to Jean Piaget's seminal work and continues to the current day (e.g., Gelman, 2003; Gopnik, 2012; Jones \& Smith, 1993). We find that rather than relying on perceptual heuristics, children from age 4 make flexible and abstract inferences when reasoning about artifacts' designs. By teasing apart these accounts, our findings shed new light on these fundamental questions about the nature of children's reasoning.

\section{Acknowledgments}

We thank Monica Alcasid, Breanna Bowers, Alexis Dawson, Vanessa Dominguez, Olivia Gerald, Carissa Jantz, Emma Kim, Renee Landin, Nora Lyang, Saloni Patel, Elizabeth Megan Scott, Kaitlyn Seifert, Mai $\mathrm{Vu}$, and Michelle Yasavolian for their assistance with stimulus design and data collection. This material is based upon work supported by the National Science Foundation Grant No BCS-1749551 to AS.

\section{References}

Baker, C.L., Jara-Ettinger, J., Saxe, R., \& Tenenbaum, J.B. (2017). Rational quantitative attribution of beliefs, desires, and percepts in human mentalizing. Nature Human Behavior, 1, 1-10.

Baker, C.L., Saxe, R., \& Tenenbaum, J.B. (2009). Action understanding as inverse planning. Cognition, 113, 329349.

Bloom, P., \& Markson, L. (1998). Intention and analogy in children's naming of pictorial representations. Psychological Science, 9, 200-204.

Dennett, D.C. (1987). The intentional stance. Cambridge: MIT Press.

Dennett, D.C. (1990). The interpretation of texts, people and other artifacts. Philosophy and Phenomenological Research, 50, 177-194.

Diesendruck, G., Markson, L., \& Bloom, P. (2003). Children's reliance on creator's intent in extending names for artifacts. Psychological Science, 14, 164-168.

Gelman, S.A. (2003). The essential child: Origins of essentialism in everyday thought. Oxford: Oxford University Press.

Gelman, S.A., \& Bloom, P. (2000). Young children are sensitive to how an object was created when deciding what to name it. Cognition, 76, 91-103.

Gergely, G., Nádasdy, Z., Csibra, G., \& Bíró, S. (1995). Taking the intentional stance at 12 months of age. Cognition, 56, 165-193.

Gopnik, A. (2012). Scientific thinking in young children: Theoretical advances, empirical research, and policy implications. Science, 337, 1623-1627.

Gopnik, A., \& Sobel, D.M. (2000). Detecting blickets: How young children use information about novel causal powers in categorization and induction. Child Development, 71, 1205-1222.

Gosling, S. (2008). Snoop: What your stuff says about you. New York: Basic Books.

Harris, P.L., Yang, B., \& Cui, Y. (2017). 'I don't know': Children's early talk about knowledge. Mind \& Language, 32, 2017.

Hauser, M., \& Wood, J. (2010). Evolving the capacity to understand actions, intentions, and goals. Annual Review of Psychology, 61, 303-324.

Henrich, J. (2017). The secret of our success: How culture is driving human evolution, domesticating our species, and making us smarter. Princeton: Princeton University Press.

Hurwitz, E., Brady, T.F., \& Schachner, A. (2019). Detecting social transmission in the design of artifacts via inverse 
planning. In A. Goel, C. Seifert, \& C. Freska, Proceedings of the 41st Annual Conference of the Cognitive Science Society.

Jara-Ettinger, J., Gweon, H., Schulz, L.E., \& Tenenbaum, J.B. (2016). The naïve utility calculus: Computational principles underlying commonsense psychology. Trends in Cognitive Sciences, 20, 589-604.

Jones, S.S., \& Smith, L.B. (1993). The place of perception in children's concepts. Cognitive Development, 8, 113-139.

Kelemen, D., Seston, R., \& Saint Georges, L. (2012). The designing mind: Children's reasoning about intended function and artifact structure. Journal of Cognition and Development, 13, 439-453.

Legare, C.H., \& Nielsen, M. (2015). Imitation and innovation: The dual engines of cultural learning. Trends in Cognitive Sciences, 19, 688-699.

Lipton, P. (2004). Inference to the best explanation $\left(2^{\text {nd }} E d\right.$.). New York: Routledge.

Liu, S., Ullman, T.D., Tenenbaum, J.B., \& Spelke, E.S. (2017). Ten-month-old infants infer the value of goals from the costs of actions. Science, 358, 1038-1041.

Marsh, L.E., Ropar, D., \& Hamilton, A.F. de C. (2014). The social modulation of imitation fidelity in school-age children. PLoS ONE, 9, e86127.

McGuigan, N., \& Whiten, A. (2009). Emulation and "overemulation" in the social learning of causally opaque versus causally transparent tool use by 23 - and 30-montholds. Journal of Experimental Child Psychology, 104, 367381.

McGuigan, N., Whiten, A., Flynn, E., \& Horner, V. (2007). Imitation of causally opaque versus causally transparent tool use by 3- and 5-year-old children. Cognitive Development, 22, 353-364.

Nielsen, M., \& Tomaselli, K. (2010). Overimitation in Kalahari Bushman children and the origins of human cultural cognition. Psychological Science, 21, 729-736.

Olson, K. R., \& Shaw, A. (2011). 'No fair, copycat!': what children's response to plagiarism tells us about their understanding of ideas. Developmental Science, 14, 431439.

Over, H., \& Carpenter, M. (2015). Children infer affiliative and status relations from watching others imitate. Developmental Science, 18, 917-925.

Pesowski, M.L. \& Friedman, O. (2019). Children value objects with distinctive histories. Journal of Experimental Psychology: General, 148, 2120-2128.

Piaget, J. (1929). The child's conception of the world. London: Routledge \& Kegan Paul.

Richins, M.L. (1994). Valuing things: The public and private meaning of possessions. Journal of Consumer Research, 21, 504-521.

Schachner, A., Brady, T.F., Oro, K., \& Lee, M. (2018). Intuitive archeology: Detecting social transmission in the design of artifacts. In C. Kalish, M. Rau, J. Zhu, \& T. Rogers, Proceedings of the 40th Annual Conference of the Cognitive Science Society.
Skerry, A.E., Carey, S.E., \& Spelke, E.S. (2013). First-person action experience reveals sensitivity to action efficiency in prereaching infants. Proceedings of the National Academy of Sciences, 110, 18728-18733.

Soley, G., \& Spelke, E.S. (2016). Shared cultural knowledge: Effects of music on young children's social preferences. Cognition, 148, 106-116.

Spelke, E.S., \& Kinzler, K.D. (2007). Core knowledge. Developmental Science, 10, 89-96.

Tenenbaum, J.B., \& Griffiths, T.L. (2001). Generalization, similarity, and Bayesian inference. Behavioral and Brain Sciences, 24, 629-640.

Tenenbaum, J.B., Griffiths, T.L., \& Kemp, C. (2006). Theory-based Bayesian models of inductive learning and reasoning. Trends in Cognitive Sciences, 10, 309-318.

Tomasello, M. (1999). The cultural origins of human cognition. Cambridge: Harvard University Press. 